LAWRENCE LIVERMORE N A T IO N A L LABORATORY

\title{
SIMULATION OF INTENSE BEAMS FOR HEAVY ION FUSION
}

\author{
A. Friedman
}

June 14, 2004

15th International Symposium on Heavy Ion Inertial Fusion Princeton, NJ, United States June 7, 2004 through June 11, 2004 
This document was prepared as an account of work sponsored by an agency of the United States Government. Neither the United States Government nor the University of California nor any of their employees, makes any warranty, express or implied, or assumes any legal liability or responsibility for the accuracy, completeness, or usefulness of any information, apparatus, product, or process disclosed, or represents that its use would not infringe privately owned rights. Reference herein to any specific commercial product, process, or service by trade name, trademark, manufacturer, or otherwise, does not necessarily constitute or imply its endorsement, recommendation, or favoring by the United States Government or the University of California. The views and opinions of authors expressed herein do not necessarily state or reflect those of the United States Government or the University of California, and shall not be used for advertising or product endorsement purposes. 


\title{
Simulation of intense beams for Heavy Ion Fusion
}

\author{
Alex Friedman* \\ Lawrence Livermore National Laboratory, University of California, \\ Livermore CA 94550 USA; and the Heavy Ion Fusion Virtual National Laboratory
}

(Dated: June 9, 2004)

\begin{abstract}
Computer simulations of intense ion beams play a key role in the Heavy Ion Fusion research program. Along with analytic theory, they are used to develop future experiments, guide ongoing experiments, and aid in the analysis and interpretation of experimental results. They also afford access to regimes not yet accessible in the experimental program. The U.S. Heavy Ion Fusion Virtual National Laboratory and its collaborators have developed state-of-the art computational tools, related both to codes used for stationary plasmas and to codes used for traditional accelerator applications, but necessarily differing from each in important respects. These tools model beams in varying levels of detail and at widely varying computational cost. They include moment models (envelope equations and fluid descriptions), particle-in-cell methods (electrostatic and electromagnetic), nonlinear-perturbative descriptions (" $\delta \mathrm{f}$ "), and continuum Vlasov methods. Increasingly, it is becoming clear that it is necessary to simulate not just the beams themselves, but also the environment in which they exist, be it an intentionally-created plasma or an unwanted cloud of electrons and gas. In this paper, examples of the application of simulation tools to intense ion beam physics are presented, including support of present-day experiments, fundamental beam physics studies, and the development of future experiments. Throughout, new computational models are described and their utility explained. These include Mesh Refinement (and its dynamic variant, Adaptive Mesh Refinement); improved electron cloud and gas models, and an electron advance scheme that allows use of larger time steps; and moving-mesh and adaptive-mesh Vlasov methods.
\end{abstract}

PACS numbers: 29.27.-a; 52.65.Cc; 52.65.Rr

Keywords: Accelerator, Fusion, Heavy-ion, Induction, Simulation, Particle-in-cell, Plasma, Beam

\section{INTRODUCTION}

In Heavy Ion Fusion (HIF) beam research [1] a number of simulation efforts are in progress, using a variety of simulation methods that include new and innovative capabilities. This paper describes some of the applications and methods being pursued in the U.S. program, in some cases in collaboration with other programs.

Particle-in-cell (PIC) methods have been the workhorses of this effort, because most of the phenomena of interest are kinetic in nature; nonetheless, moment methods remain useful for rapid scoping and exploration. The WARP code (not an acronym) incorporates both classes of model, and is used heavily in HIF beam studies [2]. WARP offers 3D Cartesian, axisymmetric $(r, z)$, and transverse $(x, y)$ "slice" geometries; at present it incorporates an electrostatic model for the space-charge field, and uses an explicit particle advance. It is especially well suited for injector studies, and for simulation of longdistance beam acceleration and transport in the presence of complex applied fields, bends (the capability which gave the code its name), and internal beam line structures. Current thrusts include the development of a detailed set of models for stray electrons ("electron cloud") and gas; development of increasingly capable Adaptive

\footnotetext{
${ }^{*}$ Correspondence to: Dr. Alex Friedman, Mail Stop 47R0112, Lawrence Berkeley National Laboratory, One Cyclotron Road, Berkeley CA 94720; 510-486-5592; mailto:af@llnl.gov
}

Mesh Refinement for the field solution; and incorporation of a plasma ion source modeling capability.

The LSP code ("Large Scale Plsmas") is also PICbased [3]. It incorporates a hybrid model (representing some components of the plasma via a particle-based fluid description). It is fully electromagnetic and implicit, and offers multiple 3D and 2D geometries (Cartesian, cylindrical, and most recently spherical). Models for collisions, ionization processes, external "ports" for radiation, and particle emission are included. It is well suited to problems involving plasmas; even dense plasmas can be handled efficiently via the hybrid implicit capability.

A development of the PIC method, nonlinear perturbative (" $\delta \mathrm{f} ")$ simulation, uses the particles as markers to evolve the difference between the current value of the distribution function and a reference (typically equilibrium) value. Widely used for stationary magnetically confined plasmas, the method has been adapted to the simulation of beams, and embodied in BEST ("Beam Equilibrium, Stability, and Transport") [4]. The technique offers reduced noise relative to conventional PIC and has been especially successful in revealing the structure and behavior of beam oscillation modes.

Vlasov methods advance the distribution function on a grid in phase space, and should be well suited for halo problems because of the very large dynamic range in phase space density that they offer. Advances in these methods are summarized herein; for details see $[5,6]$.

This paper is organized as follows. Section II describes the application of simulation tools to ongoing experiments: injector experiments on the LLNL Source Test 
Stands (STS-100 and STS-500) and on the UMER facility at the University of Maryland (and the use of AMR in these studies); the High Current Experiment (HCX) at LBNL; and the Neutralized Transport Experiment (NTX) at LBNL. This work includes experimental design and scoping, simulations in support of machine operation, code benchmarking, and related activities. Section III describes some fundamental issues in beam science and the simulation efforts being applied in their exploration: electron cloud and gas (and new models and methods under development for this topic); the effects of quadrupole strength errors; beam instabilities; and the generation of halo (and the promise of Vlasov methods for such studies). Section IV describes the application of simulations to future experiments: an Integrated Beam Experiment (IBX) and a full-scale fusion system as embodied in the Robust Point Design (RPD) study; and a possible sequence of Neutralized Drift Compression Experiments (NDCX's) culminating in a Modular Driver system. Concluding comments are offered in Section V.

\section{PRESENT-DAY EXPERIMENTS}

INJECTORS (STS AND UMER) - Injector diode calculations have long suffered from errors associated with insufficient spatial resolution in the field mesh; use of a uniform mesh fine enough to represent the regions around the emitting surface and the edge of the beam led to calculations that were too memory-intensive and timeconsuming for iterative use. Recently, a major new computational capability, the merger of Mesh Refinement (MR) and its adaptive generalization (AMR) with PIC methods, has become available [7, 8]. WARP currently incorporates an axisymmetric $(r, z)$ AMR package, and a linkage to the general-purpose 3D AMR package Chombo is working in prototype. For the HCX injector, routine steady-state simulations are accelerated by factors of 4 10. Larger payoffs have been achieved in studies of the current-rise waveform achieved in reduced-voltage experiments on STS-500, studying effects that limit the rapidity of that rise; in some cases, mesh refinement factors of up to 1000 were used.

WARP was used to model the high-voltage operation of the STS-500 diode; good agreement between simulated and measured transverse phase space distributions was obtained [9-11]. In addition, simulations of the UMER electron gun were carried out, in an effort to understand the velocity-space distribution of the emitted beam, measured by passing the beam through a pinhole and viewing the image on a phosphor screen $[12,13]$.

An alternative approach to a heavy-ion injector consists of merging a large number $(\sim 100)$ of small beamlets after they have been accelerated in tandem through a set of aperture lenses. The goal is to obtain both high current and high current density, in a transverse form factor no larger than that of the transport system, so that an ultimate multi-beam injector need not have a "dandelion" configuration. A series of experiments exploring the relevant physics is being carried out on the STS facilities, with heavy reliance on WARP simulations of the entire system $[9,11,14]$. Some of the simulation output for the final STS-500 configuration (119 beamlets, 0.07 A, $400 \mathrm{keV}$ final energy) is shown in Fig. 1; another view is shown in [11].

High-CurRent ExPeriment (HCX) - Using WARP's internal envelope equation solver and an optimization algorithm (implemented in the code's Python-based "code steering" capability), a very large number of envelope calculations, including centroid offsets, were carried out in order to "match" the HCX beam into the 4-quadrupole magnetic transport line, in which the lattice period is much longer than in the upstream electrostatic lattice. This yielded an approximate operating point with a smooth envelope that minimized the maximum transverse extent of the beam; then WARP "slice" simulations in $(x, y)$ geometry were carried out to see the deviation of the extreme-particle edge from the envelope, and the matching re-optimized. Parametric variations (in both experiment and simulations) with small changes in magnet gradients and clearing electrode biases were conducted. It appears that sufficient clearance has been achieved that the inserted electron-cloud and slow-ion diagnostics will not suffer direct beam impacts.

During the course of this work, it was noted that rapid beam shape changes, especially those involving beams far from circularity in cross-section, can cause considerable emittance growth, though quantitative agreement between simulations and experiments is still being pursued [15]. (Indeed, this effect had been seen earlier, for example in Fig. 3 of [16], but was not well understood). The observation motivated a more fundamental simulation- and theory-based study of emittance growth associated with the collective relaxation of space-charge nonuniformities [17].

Simulations initiated with beam particle distributions synthesized tomographically from measured data at the upstream end of the electrostatic transport line were carried out, using the methods described in [16]. To date, starting with the three measured 2D projections $f(x, x \prime)$, $f\left(y, y^{\prime}\right)$, and $f(x, y)$, we have obtained coarse agreement with the measured downstream distribution (see Fig. 2). The low-order moments are similar, but, while both simulated and measured spatial distributions exhibit "hollowing," the measured distribution has taken on a more pronounced diamond shape. It is conjectured that the discrepancy arises from an imperfect initial distribution which could not take into account more recently observed [18] correlations in "other" planes, e.g. $(y, x \prime)$. Initialization using data from new "optical" diagnostics [18] that yield the full $4 \mathrm{D}$ transverse phase space, or $3 \mathrm{D}$ projections thereof, may yield better agreement.

Neutralized Transport Experiment (NTX) - The NTX experiment was designed, characterized, and is be- 
ing run with the support of simulations $[19,20]$. The NTX beam, from source through the final optic, was simulated with WARP to explore the variation of the converging beam properties upon the quadrupole strengths. The observed dependence of the beam image upon the strengths of the quadrupole magnets was in good agreement with simulation results [21].

The main aim of the experiment is to study the neutralization of a beam and its focusing to a waist. For these studies, the workhorse code has been LSP. In one mode of operation, LSP is initialized with the output of WARP; in another, measured 4D phase space data (obtained by passing the beam though a movable hole created by crossed slits, then imaging it on a scintillator [18]) is used to more definitively set the initial conditions for the LSP run. An image from one simulation is shown in Fig. 3; this run used 3D cylindrical geometry with 8 azimuthal spokes, and included both a $3-\mathrm{eV}$ "plug" plasma with density $3 \times 10^{9} \mathrm{~cm}^{-3}$ and a volume plasma with density $10^{10} \mathrm{~cm}^{-3}$; it ran for two days on four PC processors. These model chamber transport simulations are in good agreement with the NTX experiments [21, 22].

\section{FUNDAMENTAL BEAM SCIENCE}

Electron Cloud and Gas - The interactions of positive-charge particle beams with stray electron "clouds" and gas in the beam line are increasingly being recognized as concerns in a variety of accelerator applications, and HIF is proving to be no exception. The fields of an uncontrolled population of electrons can deflect the beam, or induce a mismatch leading to halo production and particle loss. Because HIF beams need high line charge densities, electrons are readily trapped in the beam potential (those created by beam-induced ionization of gas are "born trapped"), and because there is an economic mandate to use as large a beam-pipe filling factor as possible (especially in a multi-beam system), a quantitative understanding of the sources, sinks, and dynamics of stray electrons is essential. Ion induction accelerators for HIF differ in key regards from other accelerators. In particular, at low energy a large fraction of the lattice is taken up by focusing magnets, traditionally assumed to be quadrupoles, though solenoids are of increasing interest; the beam pipes in these magnets are hard to clean well, leading to relatively large desorption coefficients; and accelerating gaps interact with the electrons.

A program for modeling these effects has been initiated, including the development of new simulation capabilities and the application of simulation tools to explore the relevant issues, at first singly and then in a fully integrated and benchmarked manner. A roadmap is shown in Fig. 4. To date, studies have used WARP to examine the effects of a wide variety of assumed electron cloud distributions on ion beams, and have linked ionbeam simulation, wall-desorbed electron generation, and electron tracking to estimate the electron cloud resulting from beam halo-particle loss. This work is described in [23]; see also [24].

As part of this effort, an algorithm was developed for computing trajectories of electrons using time steps similar to those used for the beam ions, as the electrons move through regions wherein they may be unmagnetized, strongly magnetized, or in an intermediate regime; see [23]. This algorithm "interpolates" between the advanced-time coordinates obtained by computing the full electron dynamics (via the usual Lorentz force mover) and those obtained by means of drift equations. This new algorithm is of general significance and may have application to other fields, including magnetic fusion, astrophysics, and near-space physics.

QuAdrupole Strength ERrors - A concern exists about the cumulative effect of random errors in the strength of quadrupole magnets used for beam confinement. To address this, a series of $\operatorname{WARP}(x, y)$ simulations was carried out [25]. These showed that, over the 200 lattice periods simulated, uniform-random strength errors of $0.1 \%$ induce only small emittance growth in a driver-like beam with phase advance depressed by a factor of ten due to space charge. Mismatch oscillations are seen to grow in a random-walk manner, and emittance growth occurs primarily as halo generation. Confining the mismatch to less than about $15 \%$ ensures minimal emittance growth; larger mismatch amplitudes can induce serious growth. In Fig. 6, the results of an ensemble of 14 WARP runs are shown, and the evolution of the emittance compared with a scaling rule that bounds the growth by assuming continuous thermalization of mismatch energy [26]. This scaling seems pessimistic, but the simulations need to be extended to a thousand lattice periods or more.

Instabilities - Considerable theoretical and computational study is being applied to the various possible unstable modes [27-29], which include anisotropy driven modes, two-stream interactions, resistive wall-like modes driven by coupling to the accelerating module impedance [30], and others.

One mode of special concern is an electrostatic temperature anisotropy-driven mode. When $T_{\perp}>T_{\|}$, free energy is available for a Harris-like instability. First discovered in 1990 in WARP 3-D simulations [31] and studied with further PIC simulations [32], this mode has recently been examined in detail using BEST [33]. That work confirmed that the mode saturates quasilinearly before equipartitioning, with final $\Delta v_{\|} \approx \Delta v_{\perp} / 3$. These studies show that driver designs must take this effect into account. BEST was also applied to Weibel; that mode appears unimportant for energy isotropization.

Simulation studies of the electron-ion two-stream instability using BEST show that the instability has a characteristic dipole-mode structure, and that its threshold decreases with increasing fractional neutralization and 
increases with increasing axial momentum spread of the beam particles. In the nonlinear phase, the simulations show that the instability first saturates at a relatively low level, and subsequently grows to a higher level [34]. LSP is also being applied to the two-stream instability [35], and it is planned that WARP will be studying the mode as well, using the new electron-cloud models described above.

HALO - Vlasov methods which advance the distribution function $f(\mathbf{x}, \mathbf{v})$ on a grid in phase space have been applied very effectively to problems in longitudinal beam dynamics [36]. They offer great promise for halo problems because they naturally can represent a very large dynamic range in $f$, in contrast with PIC methods which require many particles to accomplish this. As an example, Fig. 7 shows the evolved state of a density-mismatched axisymmetric thermal beam with phase advance depressed to half its single-particle value, as computed using a prototype Vlasov solver for the $4 \mathrm{D}$ transverse phase space evolution. However, until recently these methods have been unsuitable for beams in quadrupole channels because of the rapid oscillatory motion through phase space in such systems, requiring a very large mesh and suffering from excessive flow through that mesh. New methods, using moving grids and/or an adaptive mesh, promise to dramatically improve the efficiency of Vlasov methods on such problems $[5,6]$.

\section{FUTURE EXPERIMENTS}

Integrated BeAm ExPERIMENT (IBX) AND RoBUst Point Design (RPD) - The most well-developed approach to an HIF system is based on non-neutral drift compression and final focusing, and neutralized-ballistic chamber transport. Two studies examined such systems, the first in the context of a possible next-step IBX, the second in the context of a full-scale RPD. Both systems were studied using theory and simulation, in consultation with researchers carrying out system studies and engineering analyses.

A candidate concept for an IBX was selected for an integrated simulation study from the range of possible options [37-39]. A plausible physics design was synthesized, with a model injector, a focusing lattice, and a longitudinal-dynamics design including accelerating and beam-end confining ("ear") waveforms. 3D WARP simulations of this "ideal" IBX [40] follow the beam as it is created at the source, matched, accelerated, and begins to drift-compress; see Fig. 8, which shows the line-charge density at 100 successive times. The beam head and body exhibit quiescent behavior; the tail, which was originally emitted at late time, separates cleanly and is left behind by the accelerating beam.

Initial 3D WARP simulations of drift compression in an IBX with 10:1 ratio [41] have been carried out (the evolution of the longitudinal phase space in one such run is shown in Fig. 9). These runs show some transverse emittance growth and loss to halo; it is expected that this will improve with more optimal pulse-shaping.

Considerable progress has been made in understanding compression, focusing, and chamber transport at driver scale. Much, but not all, of this work is associated with the development of a self-consistent RPD, described in $[42,43]$. Studies of pulse compression for an RPD are described in [41]. Chamber transport simulations also played an important role in establishing the constraints on focusing angle, ion mass and energy, and other parameters for the RPD, and in highlighting the special attention that must be given to the early-time "foot" beams $[44,45]$. The latter, in contrast with the mainpulse beams, do not have the benefit of neutralizing electrons created via photoionization of background gas by $\mathrm{x}$-rays from heated targets. Output from a pair of LSP simulations of an RPD main pulse ( $\left.2 \mathrm{kA}, 4 \mathrm{GeV}, \mathrm{Bi}^{+}\right)$ in the chamber is presented in Fig. 10. In the absence of neutralization, focusing is very poor; with neutralization, a focal spot with $1.2 \mathrm{~mm}$ RMS radius is achieved.

\section{Neutralized Drift COMPRESSION ExpERIMENTS} (NDCX's) AND Modular DRIVER - Solenoid lenses, in comparison with quadrupoles, offer to transport a highline-charge beam at low kinetic energy [48]. However, they impose the challenge of creating such a beam. One approach to the generation of such a beam is an "acceldecel" sequence that overrides the Child-Langmuir dependence of current upon diode voltage. This process is being studied using WARP and other tools [49].

Neutralized drift compression offers the promise of a large compression ratio obtained using a short drift length; however, it imposes the requirement of a final focusing system with a very large chromatic acceptance. LSP simulations show that a solenoid field can focus an ion beam with a $20 \%$ head-to-tail energy variation ("tilt") in a plasma, as it continues to drift compress. A sequence of experiments using modified NTX equipment, called the Neutralized Drift Compression Experiments (NDCX's), is being analyzed; it will explore all of these processes at modest scale [1]. One step in the NDCX sequence envisions using 0.5 to $1 \mathrm{MeV} \mathrm{He}^{+}$ions; given 0.1 percent velocity control, LSP simulations show the beam compressed to a $\sim 1 \mathrm{~ns}$ duration and $1 \mathrm{~mm}$ radius as it impinges on a target [22].

LSP is being used in planning for the NDCX's, and for studies of the basic physics of neutralized drift compression, including the transition from unneutralized Brillouin flow in solenoids to neutralized drift compression (both the neutralization process and the prevention of premature neutralization, using a dipole to suppress upstream electron flow), filamentation (largely suppressed by the magnetic field), two-stream instability (which 1D simulations show to be benign), and final focus in a modular solenoid accelerator configuration using plasma lenses [22]. Analysis, and simulation using the 2D electromagnetic code EDPIC assuming a "frozen" beam and 
background ions, examined the launching of electron waves by a beam immersed in a solenoid field [50].

The Modular Driver approach is being explored though the synthesis and analysis of a Modular Point Design [43]. Initial integrated LSP simulations of neutralized compression and focusing in a $100-\mathrm{m}$ plasma column, using a conductivity model to approximate the response of a plasma, predict $>90 \%$ transport efficiency to within the 5-mm spot needed for a hybrid distributed radiator target, even though some filamentation is present [22]; see Fig. 11.

\section{DISCUSSION}

The work summarized herein shows how the HIF beam simulation effort is evolving toward "multiphysics, multiscale" modeling. To simulate electron cloud and gas effects, new models (including source models adapted from high-energy physics modeling) are being implemented in WARP, as is a new method for bridging disparate electron and ion time scales. Plasma interactions are of increasing interest, and LSP, which already includes implicit hybrid capabilities as well as collisional and ionization effects, is being applied more widely. Injectors, especially those of the merging-beamlet type, are inherently "multiscale," and plasma-based sources are of in- creasing interest. Finally, the new high energy density physics mission of the U.S. program, and a complementary recognition of the benefits of a modular driver, are motivating new experimental and simulation plans.

While this paper emphasizes simulations, analytic theory also plays an important role in HIF research (roughly one-third of the combined simulation-theory effort). Even for simulations, this paper does not cover every element of the U.S. program, nor does it attempt to cover related work elsewhere. Other notable algorithmic developments not described herein include a new, single-pass implicit Maxwell equation solver recently implemented in LSP [51], and studies of new Darwin (magnetoinductive) methods for use in BEST and other codes [52]. Another notable application is 3D WARP simulation of the Paul Trap Experiment [53].

\section{Acknowledgments}

The work of a number of people formed the basis for this paper; the References provide a partial list. This work was performed under the auspices of the U.S. D.o.E. by the University of California, Lawrence Livermore National Laboratory under Contract No. W-7405-ENG-48.
[1] B. G. Logan, et. al., these Proceedings.

[2] D. P. Grote, A, Friedman, I. Haber, and S. S. Yu, Fusion Eng. and Design 32-33, 193 (1996); D. P. Grote, et. al., Nucl. Instr. and Meth. Phys. Res. A 464, 563 (2001).

[3] T. P. Hughes, S. S. Yu, and R. E. Clark, Phys. Rev. Spec. Topics - Accel. and Beams 2, 110401 (1999); D. R. Welch, D. V. Rose, B. V. Oliver, and R. E. Clark, Nucl. Instr. and Meth. Phys. Res. A 464, 134 (2001).

[4] H. Qin, R. C. Davidson, and W. W. Lee, Phys. Rev. Spec. Topics - Accel. and Beams 3, 084401 (2000).

[5] E. Sonnendrucker, A. Friedman, J. J. Barnard, D. P. Grote, and S. M. Lund, Nucl. Instr. and Meth. Phys. Res. A 464, 470 (2001).

[6] E. Sonnendrucker, et. al., these Proceedings.

[7] J.-L. Vay, et. al., Phys. Plasmas 11, 2928 (2004).

[8] J.-L. Vay, et. al., these Proceedings.

[9] J. W. Kwan, et. al., "Ion Source and Injector Experiments at the HIF VNL," these Proceedings.

[10] J. W. Kwan, et. al., "Production of a High Brightness Beam from a Large Surface Source," these Proceedings.

[11] G. Westenskow, et. al., these Proceedings.

[12] I. Haber, et. al., these Proceedings.

[13] R. A. Kishek, et. al., these Proceedings.

[14] D. P. Grote, E. Henestroza, and J. W. Kwan, Phys. Rev. Spec. Topics - Accel. and Beams 6, 014202 (2003).

[15] L. R. Prost, et. al., these Proceedings.

[16] A. Friedman, D. P. Grote, C. M. Celata and J. W. Staples, Laser and Particle Beams 21(1), 17 (2003).

[17] S. M. Lund, et. al., these Proceedings.

[18] F. M. Bieniosek, et. al., these Proceedings.
[19] E. Henestroza, et. al., "Design and Characterization of a Neutralized-Transport Experiment for Heavy-Ion Fusion," Phys. Rev. Spec. Topics - Accel. and Beams, to be published (2004).

[20] P. K. Roy, "Results on Intense Beam Focusing and Neutralization from the Neutralized Beam Experiment," Phys. Plasmas Special Issue, to be published (2004).

[21] P. K. Roy, et. al., these Proceedings.

[22] D. R. Welch, et. al., these Proceedings.

[23] R. H. Cohen, et. al., these Proceedings.

[24] P. H. Stoltz, et. al., these Proceedings.

[25] S. M. Lund, private communication (2003).

[26] S. M. Lund, J. J. Barnard, and E. P. Lee, Proc. 2000 Linac Conf., paper MOE11, arXiv:physics/0009095.

[27] R. C. Davidson, "Survey of Collective Instabilities and Beam-Plasma Interactions in Intense Heavy Ion Beams," submitted to Phys. Rev. Spec. Topics - Accel. and Beams.

[28] H. Qin, Phys. Plasmas 10, 2078 (2003).

[29] R. C. Davidson, et. al., these Proceedings.

[30] D. A. Callahan, A. B. Langdon, A. Friedman, and I. Haber, J. Appl. Phys. 81, 2298 (1997).

[31] A. Friedman, et. al., Proc. 2nd European Part. Accel. Conf., Nice, France, P. A. Martin, Ed. (Editions Fronters, Gif-sur-Yvette, France), 2, 1699 (1990);

A. Friedman, D. P. Grote, and I. Haber, Phys. Fluids B 4, 2203 (1992).

[32] I. Haber, et. al., Fusion Eng. Design 32-33, 169 (1996); I. Haber, et. al., Nucl. Instr. and Meth. Phys. Res. A 415, 405 (1998).

[33] E. A. Startsev, Phys. Rev. Spec. Topics - Accel. and 
Beams 6, 084401 (2003);

E. A. Startsev, et. al., these Proceedings.

[34] H. Qin, E. A. Startsev, and R. C. Davidson, Phys. Rev. Spec. Topics-Accel. and Beams 6, 014401 (2003).

[35] D. V. Rose, et. al., these Proceedings.

[36] O. Boine-Frankenheim and I. Hofmann, Phys. Rev. Spec. Topics-Accel. and Beams 3, 104202 (2000).

[37] J. J. Barnard, et. al., Laser and Particle Beams 21, 553 (2004).

[38] C. M. Celata, et. al., "The Integrated Beam Experiment - A Next Step Experiment for Heavy Ion Fusion," Laser and Particle Beams, in press (2004).

[39] M. A. Leitner, et. al., these Proceedings.

[40] D. P. Grote, et. al., these Proceedings.

[41] W. M. Sharp, et. al., these Proceedings.

[42] S. S. Yu, et. al., Fusion Science and Tech. 44, 266 (2003).

[43] S. S. Yu, et. al., these Proceedings.

[44] W. M. Sharp, et. al., Phys. Plasmas 10, 2457 (2003).

[45] J. J. Barnard, et. al., these Proceedings.

[46] W. M. Sharp, et. al., Fusion Sci. Technol. 43, 393 (2003).

[47] W. M. Sharp, et. al. "Chamber Transport Modeling for Heavy-Ion-Fusion Drivers," Nucl. Fusion (2004, to be published).

[48] E. P. Lee, these Proceedings.

[49] E. Henestroza, et. al., these Proceedings.

[50] I. Kaganovich, these Proceedings.

[51] D. R. Welch, et. al., "Implementation of a Non-Iterative Implicit Electromagnetic Field Solver for Dense Plasma Simulation," Comp. Phys. Comm., to appear (2004).

[52] W. W. Lee, et. al., these Proceedings.

[53] E. P. Gilson, et. al., these Proceedings.
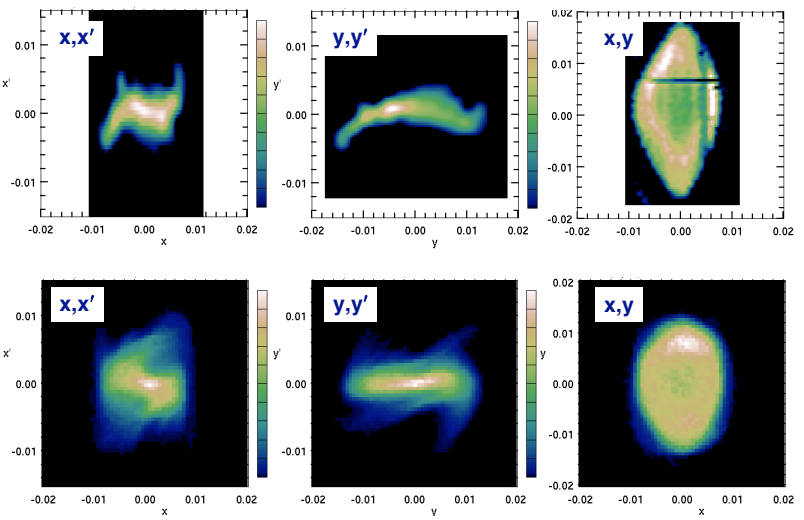

FIG. 2: Beam particle distributions at exit of HCX electrostatic lattice: upper row measured, lower row simulated.

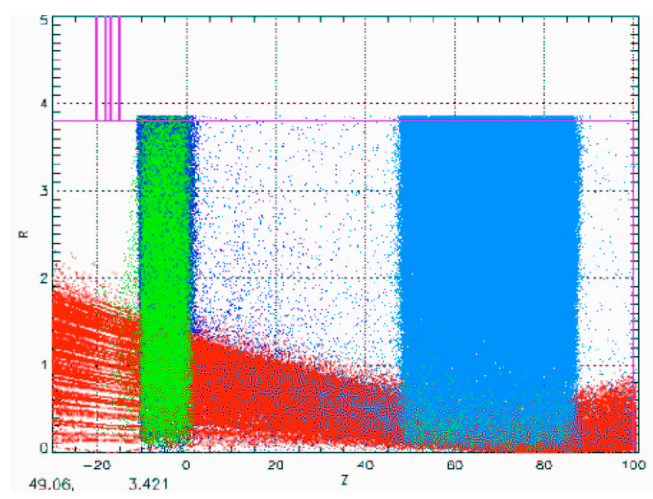

FIG. 3: LSP simulation of NTX beam, showing plug and volume plasmas.

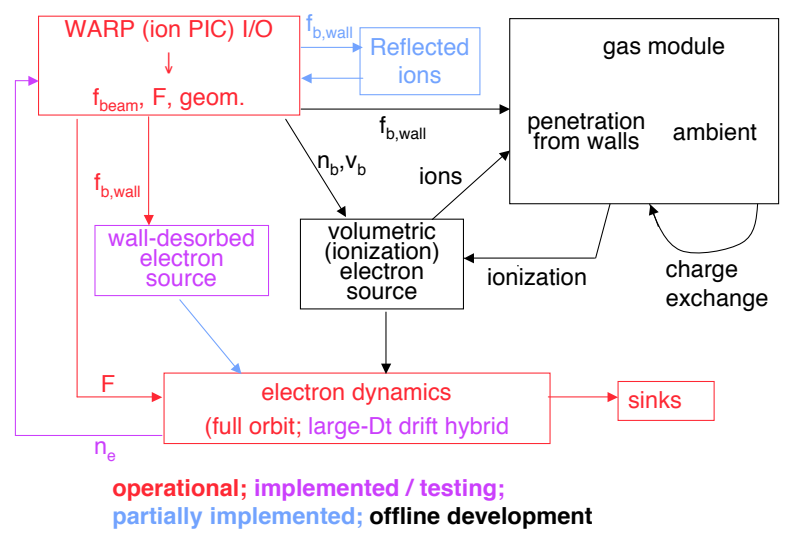

FIG. 4: "Roadmap" for self-consistent beam simulation including effects of electron cloud and gas.
FIG. 1: 3D WARP simulation of planned merging-beamlets
experiment on STS-500: (a) side view $(z, x)$; (b) phase space

FIG. 1: 3D WARP simulation of planned merging-beamlets
experiment on STS-500: (a) side view $(z, x)$; (b) phase space $(x, x) ;(\mathrm{c})$ evolution of $(x, x \prime)$ normalized emittance.

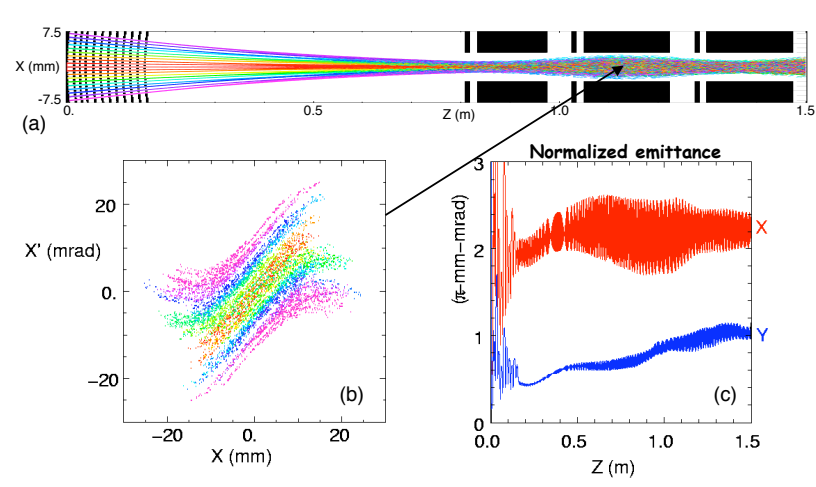




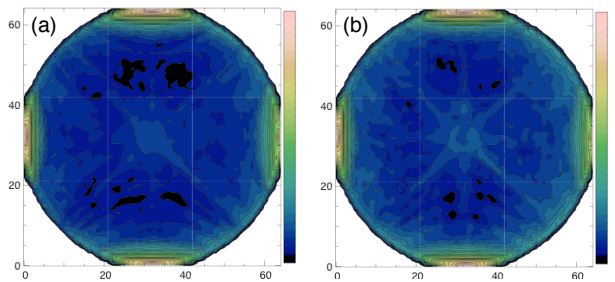

FIG. 5: Wall-desorbed electron density (logarithmic scale) as simulated in WARP; electrons in 45-degree regions are caused by first-flight reflected ions: (a) full orbit, $\Delta t=0.25 / f_{c e}$; (b) interpolated mover, $\Delta t 25$ times larger.
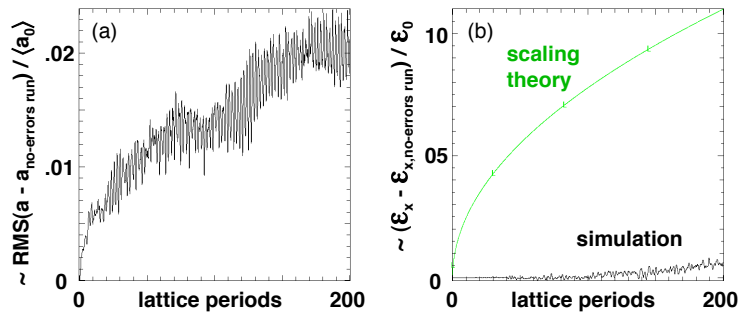

FIG. 6: Results of ensemble of 14 WARP runs with $0.1 \%$ random quadrupole strength errors: evolution of (a) relative RMS mismatch amplitude; (b) relative emittance.

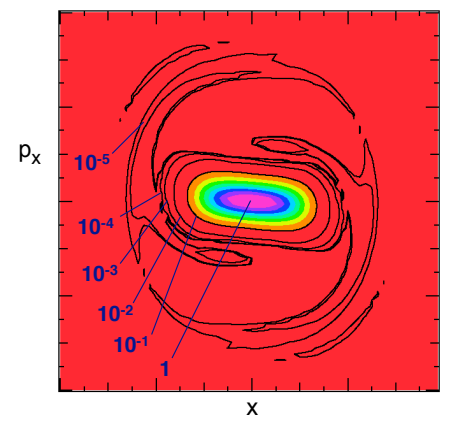

FIG. 7: Phase space density from prototype Vlasov simulation, showing halo structure.

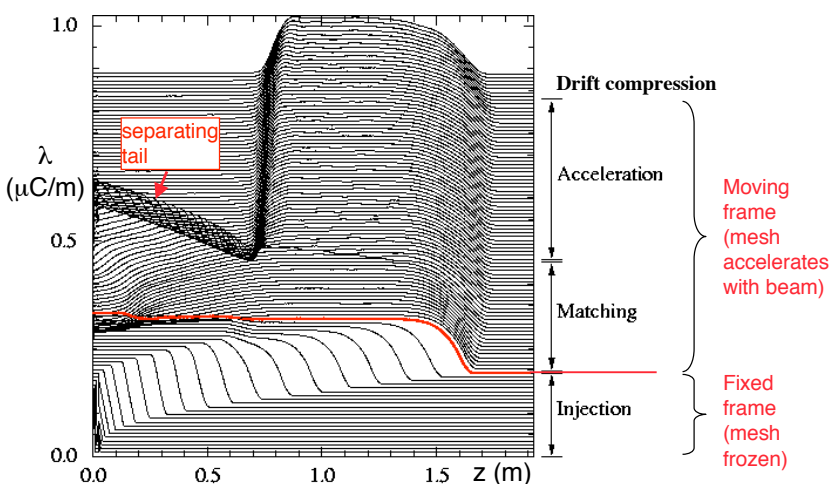

FIG. 8: 3D WARP simulation of an "ideal" IBX: line-charge at 100 successive times (vertically offset).
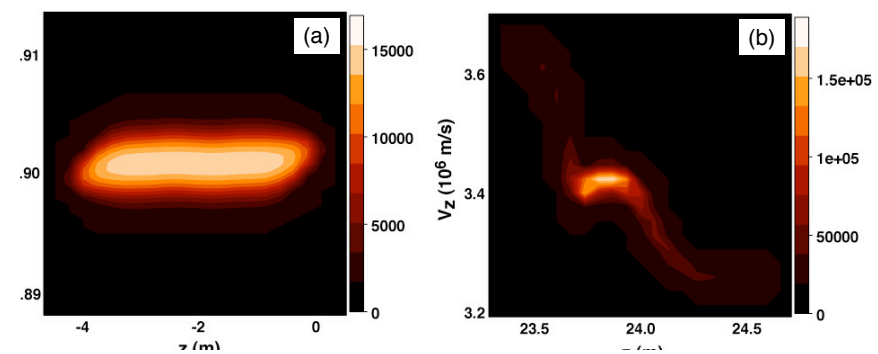

FIG. 9: 3D WARP simulation of IBX-scale drift compression: (a) initial; (b) near stagnation (note change of scale).
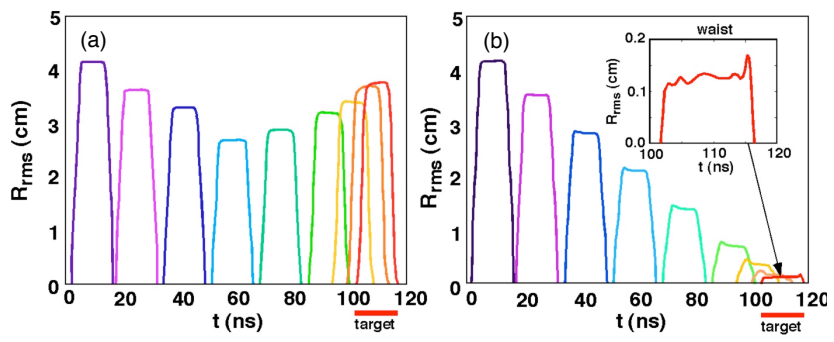

FIG. 10: Focusing of RPD-like beam as simulated using LSP; radius vs. time at selected points over a 6-m focal length: (a) no plasma neutralization; (b) with neutralization.

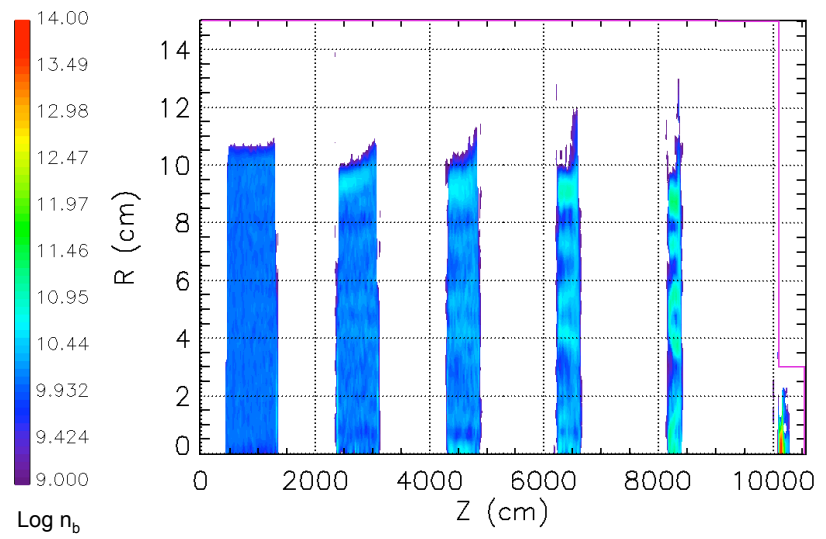

FIG. 11: LSP simulation of neutralized compression and focusing in model Modular Driver beam. 